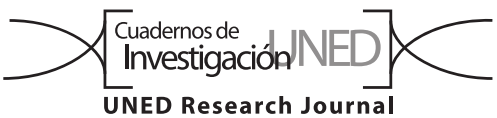

\title{
Calidad física y química de cuerpos de agua ribereños en la zona costera de Bahía Ballena y Puerto Cortés, Costa Rica
}

\author{
Juan Carlos Villegas-Arguedas \\ Universidad Nacional, Doctorado en Ciencias Naturales para el Desarrollo, Heredia, Costa Rica; juan.villegas@sinac.go.cr \\ Recibido 02-X-2018 • Corregido 10-XII-2018 • Aceptado 14-I-2019
}

\begin{abstract}
Physical and chemical quality of riparian water bodies in the coastal zone of the Ballena Bay and Cortés Port, Costa Rica". Introduction: various physical and biological factors affect coastal aquatic systems. Objective: to determine the physico-chemical conditions of four surface water bodies in Costa Rica. Methods: at each monitoring station the in situ value of the physical-chemical variables of the water was determined with a Thermo Scientific multi-parameter portable meter. Turbidity (Secchi), type of environment and current were categorized as "dummy" variables. Results: I took a total of 22 samples. Dissolved oxygen ranged b etween $8,73 \mathrm{mg} / \mathrm{L}$ in Ballena Creek and $12,88 \mathrm{mg} / \mathrm{L}$ in Coronado River; oxygen saturation from $137,2 \%$ in Tortuga River to $164,2 \%$ in Coronado River. Water temperature ranged from $25,20^{\circ} \mathrm{C}$ in Ballena Stream and $34,10^{\circ} \mathrm{C}$ in the Morete River; salinity ranged from $0,10 \mathrm{ppt}$ in the four sampling sites to $0,20 \mathrm{ppt}$ in the Morete and Coronado rivers. Total dissolved solids ranged from $64 \mathrm{mg} / \mathrm{L}$ in Tortuga River to $173 \mathrm{mg} / \mathrm{L}$ in Coronado River. The $\mathrm{pH}$ from 6,42 in Ballena Creek to 7,67 in the Coronado River. Conclusion: There were no significant differences among these rivers and streams: they all have a similar water quality.
\end{abstract}

RESUMEN: Introducción: varios factores físicos y biológicos afectan los sistemas acuáticos costeros. Objetivo: determinar las condiciones físico-químicas de cuatro cuerpos de aguas superficiales en Costa Rica. Métodos: en cada estación de monitoreo, el valor in situ de las variables físico-químicas del agua se determinó con un medidor portátil multiparamétrico Thermo Scientific. La turbidez (Secchi), el tipo de entorno y la corriente se categorizaron como variables "dummy". Resultados: tomé un total de 22 muestras. El oxígeno disuelto osciló entre $8,73 \mathrm{mg} / \mathrm{L}$ en Ballena Creek y $12,88 \mathrm{mg} / \mathrm{L}$ en el río Coronado; saturación de oxígeno desde $137,2 \%$ en el río Tortuga a $164,2 \%$ en el río Coronado. La temperatura del agua osciló entre $25,20^{\circ} \mathrm{C}$ en el arroyo Ballena y $34,10^{\circ} \mathrm{C}$ en el río Morete; salinidad de 0,10ppt en los cuatro sitios de muestreo a 0,20ppt en los ríos Morete y Coronado. El total de sólidos disueltos varió desde $64 \mathrm{mg} / \mathrm{L}$ en el río Tortuga hasta $173 \mathrm{mg} / \mathrm{L}$ en el río Coronado. El pH de 6,42 en Ballena Creek a 7,67 en el río Coronado. Conclusión: No hubo diferencias significativas entre estos ríos y arroyos: todos tienen una calidad de agua similar.

Palabras claves: Agua, físico-química, Ballena, Cortés, Costa Rica.

Key words: Water, physical-chemical, Ballena, Cortés, Costa Rica.

Los ecosistemas acuáticos -ribereños, estuarinos o marinos- están condicionados por una intrincada asociación de factores ambientales, $\mathrm{pH}$, oxígeno disuelto, demanda bioquímica de oxígeno, temperatura, nitratos, fosfatos, sedimentos, temperatura, penetración de luz, disponibilidad y profundidad de hábitat, cobertura forestal de riveras, nivel del agua, velocidad de corriente, caudal, gradientes topográficas, altitud y tipo de sustrato (Glaser \& Glaser, 1996; Posada, Roldán, \& Ramírez, 2000; Ocón \& Rodríguez, 2004), que facilitan la subsistencia de las especies.

Estos factores juegan un papel importante en la definición de los ensamblajes taxonómicos y en los conglomerados ecológicos del ecosistema (West, Boubée, \& Barrier 1997; Ramírez \& Pringle, 1998), siendo por consiguiente la química del agua superficial una alternativa para determinar el estado de conservación de estos dinámicos sistemas, medida en algún punto, el cual tendrá intrínseca sus influencias litológicas, atmosféricas, climáticas y antropogénicas (Beita-Sandí \& Barahona-Palomo, 2010).

Los ríos reflejan fielmente el estado medioambiental de una región debido a que los mismos acarrean o son depositarios de gran cantidad de materiales o substancias que se han generado en sus alrededores a partir de las actividades humanas (industria, urbanización, deforestación, minería) o por desastres naturales (huracanes, erupciones volcánicas, terremotos), transportados (as) desde diversas distancias por distintos medios como la lluvia, y finalmente depositados en el océano (Umaña 1998; Calvo-Brenes \& Mora-Molina, 2015). 
Los ecosistemas de agua dulce en Costa Rica presentan alteraciones por la acción del hombre, sin que hasta la fecha se hayan estudiado a profundidad sus efectos sobre las comunidades que allí se encuentran. Las actividades antrópicas que mayor daño causan a los ecosistemas acuáticos son las agrícolas, la extracción descontrolada de recursos biológicos, la sedimentación, los agroquímicos, el desvío de cauces, la extracción de materiales o concesiones mineras, la desecación de humedales y hasta la contaminación por descarga de aguas negras de los grandes centros poblacionales o urbanos (MINAE, 2000; SINAC, 2007; Calvo \& Mora, 2012; Parreño, Bernal, \& Palmer, 2015).

Los ríos de la zona sur costarricense siguen siendo considerados ecosistemas complejos y desconocidos y aún falta investigación sobre el comportamiento de las poblaciones dulceacuícolas y su relación con su medio ambiente (Rojas \& Rodríguez, 2008). Con la presente investigación se procuró conocer el estado actual de la calidad del agua en esta zona particular del sur de Costa Rica.

\section{MATERIALES Y MÉTODOS}

La investigación se desarrolló en la zona costera de los distritos Bahía Ballena y Puerto Cortés hasta el pie de monte, definido en la cota $20 \mathrm{msnm}$, para un área de estudio total de $12223,10 \mathrm{Ha}$, entre los $9^{\circ} 16^{\prime} 00^{\prime \prime}-8^{\circ} 7^{\prime} 00^{\prime \prime} \mathrm{N}$ \& $83^{\circ} 28^{\prime} 00^{\prime \prime}-83^{\circ} 50^{\prime} 00^{\prime \prime} \mathrm{O}$ (Fig. 1).

Se estableció en la medida de lo posible un punto de muestreo cada $10 \mathrm{~km}$ aproximadamente para un total de cuatro estaciones de muestreo (Fig. 1), 1: en el río Morete (Higuerón), 2: quebrada Ballena, 3: río Tortuga y 4: río Coronado. Se aprovechó la facilidad que brindó la carretera costanera sur para ubicar estos sitios de muestreo, por su facilidad de acceso. Las visitas a los sitios se realizaron entre junio 2017 y abril 2018 al menos una vez cada dos meses.

En cada estación de seguimiento establecida en los ecosistemas ribereños se midió el oxígeno disuelto (OD), porcentaje de saturación de oxígeno (PSO), temperatura (Temp), salinidad (Sal), sólidos disueltos totales (SDT), y la

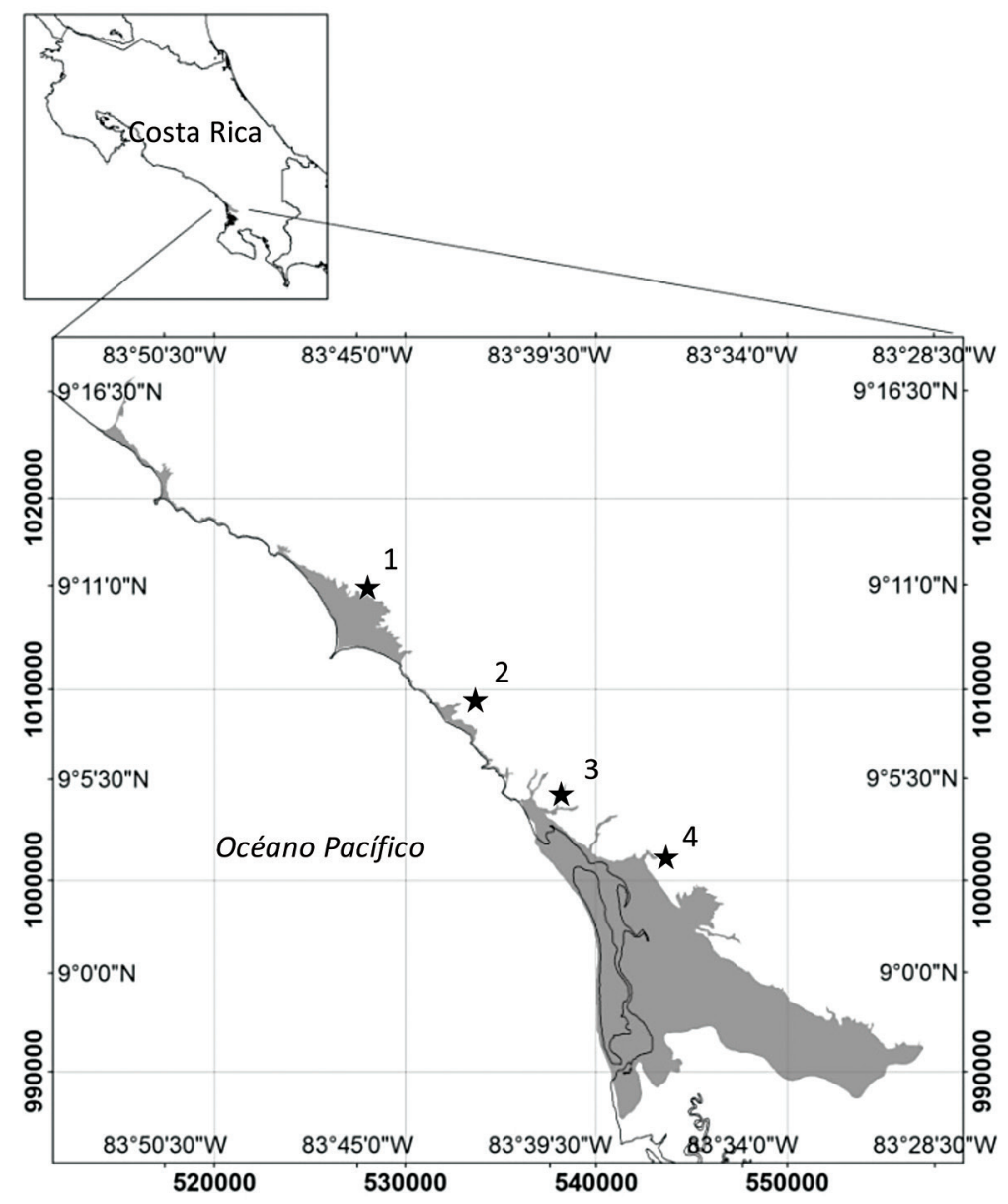

Fig. 1. Área de estudio. 1: río Morete (Higuerón); 2: quebrada Ballena; 3: río Tortuga; 4: río Coronado. 
concentración ion hidrógeno $(\mathrm{pH})$, mediante un medidor portátil multiparámetro Thermo Scientific, modelo Orion 5-Star previamente calibrado a los estándares definidos por el fabricante (Correa-Araneda et al., 2010; Aguirre, Vanegas, \& García 2016; Flores-Stulzer, Villalobos-Sandí, Piedra-Castro, \& Scholz, 2017).

Se categorizó las variables turbidez (Secchi), tipo de ambiente y corriente como variables "dummy" debido a que éstas son variables categóricas, no numéricas, y así poder utilizarlas en análisis de reducción de la multidimensionalidad estadística.

Oxígeno disuelto y porcentaje de saturación de oxígeno: estas variables se determinaron con un electrodo de membrana del medidor portátil multiparámetro Thermo Scientific con una precisión de $\pm 0,1 \mathrm{mg} / \mathrm{L}$ para el OD y $\pm 2 \%$ para el PSO, para lo cual se introdujo el electrodo en el agua y se esperó estabilización en la pantalla del instrumento para hacer la lectura del valor respectivo.

Temperatura, salinidad, sólidos disueltos totales: la cuantificación de estas variables se realizó con el electrodo de medición eléctrica del equipo multiparámetro mencionado. La lectura del registro se tomó al momento de su estabilización en la pantalla, El electrodo se utilizó para medir la concentración de SDT $( \pm 0,5 \mathrm{mg} / \mathrm{L})$, la Temp $\left( \pm 0,1^{\circ} \mathrm{C}\right)$ y Sal $( \pm 0,1 \mathrm{ppt})$ generada por la presencia de $\mathrm{NaCl}$; todos los registros se tomaron aproximadamente a los $10 \mathrm{~cm}$ de profundidad en superficie y al metro (o igual a la profundidad de muestreo) en las zonas más profundas del río o quebrada.

Concentración del ion hidrógeno $(\mathrm{pH})$ : el valor del $\mathrm{pH}( \pm 0,002)$ se estimó mediante un electrodo de cristal del equipo multiparámetro mencionado a profundidad no mayor a los $10 \mathrm{~cm}$; o a profundidad de muestreo en los sitios con poco caudal.

Turbidez (Secchi), ambiente y corriente: para la determinación de la turbidez (Secchi) del agua se utilizó un disco La Motte de $30 \mathrm{~cm}$ de diámetro, clasificándose arbitrariamente en las tres categorías definidas por Protti, Sáenz, Guevara y Herrera (2005a, 2005b, 2007): I, turbia (menor de $10 \mathrm{~cm}$ ), II, semi turbia (entre 11 y $20 \mathrm{~cm}$ ) y III, cristalina (mayor de $21 \mathrm{~cm}$ o mayor que la profundidad de muestreo), La estimación consistió en introducir el disco en el agua bajándolo lentamente por medio de una cuerda con marcas indicativas cada $5 \mathrm{~cm}$, hasta que el mismo no sea perceptible a la vista, momento en el cual se registró la respectiva profundidad.
El tipo de ambiente se categorizó arbitrariamente como: Arenoso=1; Lodoso=2; Lodoso-Arenoso=3; Rocoso $=4$ y Rocoso-Arenoso $=5$, mientras que la corriente del agua fue: Muy lenta=1, Lenta $=2 ;$ Moderada $=3$ o Fuerte $=4$.

Para determinar la significancia de las diferencias estadísticas en las variables físico-químicas del agua de los sitios de muestreo, se realizaron ANOVA no paramétricos Kruskall-Wallis según Sokal y Rohlf 1981, Fowler, Cohen y Jarvis (1998) y Umaña (1998) con la ayuda de InfoStat 1.1 2017 (Balzarini et al., 2017).

Mediante un análisis multivariado de componentes principales (Fowler, Cohen, \& Jarvis 1988; Umaña, 1998; Balzarini et al., 2017) se exploró la relación entre las variables de la calidad del agua (OD, PSO., Temp., Sal., SDT, Sech. y $\mathrm{pH}$ ), respecto a los sitios de muestreo, con la finalidad de determinar algunas asociaciones, correlaciones o similitud entre estos.

Ética, conflicto de intereses y declaración de financiamiento: el autor declara haber cumplido con todos los requisitos éticos y legales pertinentes, tanto durante el estudio como en el manuscrito; que no hay conflictos de interés de ningún tipo, y que todas las fuentes financieras se detallan plena y claramente en la sección de agradecimientos. Asimismo, está de acuerdo con la versión editada final del documento. El respectivo documento legal firmado se encuentra en los archivos de la revista.

\section{RESULTADOS}

Se registró un total de 22 muestras de la calidad de agua a partir de las variables predefinidas entre los cuatro sitios de muestreo. El oxígeno disuelto presentó valores entre los $8,73 \mathrm{mg} / \mathrm{L}$ en la quebrada Ballena y los $12,88 \mathrm{mg} / \mathrm{L}$ en el río Coronado, (Kruskall-Wallis $\mathrm{H}=1,74$; g.l. $=3 ; p=0,62$ ) (Fig. 2A). Los valores para el porcentaje de saturación del oxígeno oscilaron entre 137,2\% en el río Tortuga y $164,2 \%$ en el río Coronado (Kruskall-Wallis $H=1,33 ; g . l .=3 ; p=0,72$ ) (Fig. 2B).

La temperatura del agua registró un valor mínimo de $25,20^{\circ} \mathrm{C}$ en la quebrada Ballena y un máximo de $34,10^{\circ} \mathrm{C}$ en el río Morete, (Kruskall-Wallis $\mathrm{H}=1,89$; g.l. $=3$; $\mathrm{p}=0,59$ ) (Fig. 3A). Por su parte la salinidad presentó su valor mínimo de 0,10ppt en los cuatro sitios de muestreo y su valor máximo en los ríos Morete y Coronado con 0,20ppt, (Kruskall-Wallis $\mathrm{H}=2,93 ;$ g.l. $=3 ; \mathrm{p}=0,25$ ) (Fig. 3B). Los sólidos disueltos totales oscilaron entre un mínimo de $64 \mathrm{mg} / \mathrm{L}$ en el río Tortuga y un máximo de $173 \mathrm{mg} / \mathrm{L}$ 

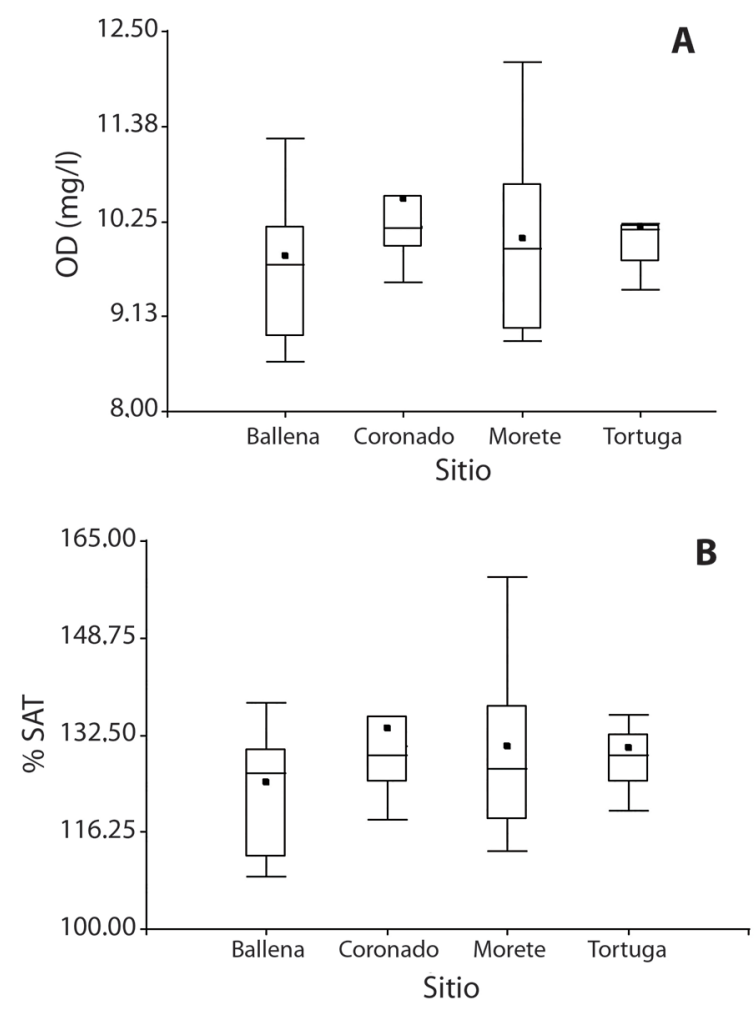

Fig. 2. Comportamiento de variables de oxígeno por sitios de muestreo (medias, medianas y error estándar) entre junio 2017 y abril 2018.

en el río Coronado (Kruskall-Wallis $\mathrm{H}=6,09 ;$ g.l. $=3$; $\mathrm{p}=0,10$ ) (Fig. 3C).

El registro de la variable concentración del ión hidrógeno tuvo un mínimo en la quebrada Ballena con 6,42 mientras que el máximo de 7,67 se registró en el río Coronado, (Kruskall-Wallis $\mathrm{H}=1,05 ; \mathrm{g} . \mathrm{l} .=3 ; \mathrm{p}=0,78$ ) sin ser tales diferencias estadísticamente significativas (Fig. 4).

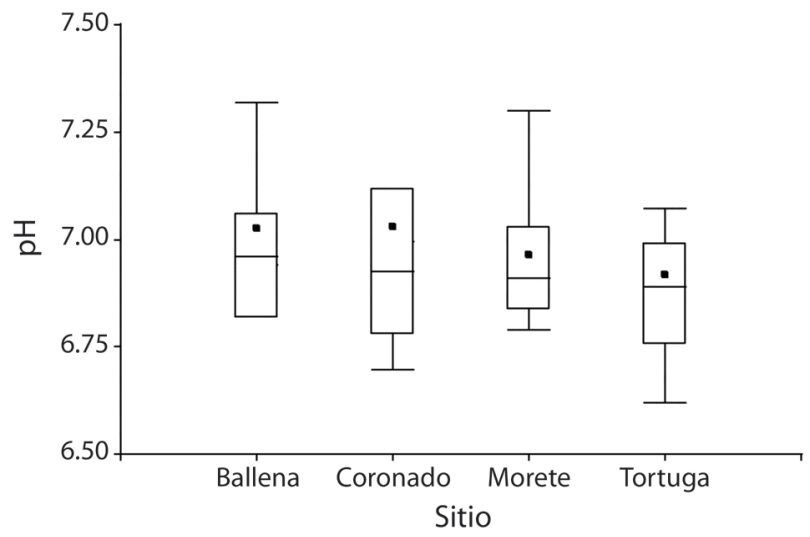

Fig. 4. Comportamiento del pH por sitios de muestreo (medias, medianas y error estándar) entre junio 2017 y abril 2018.
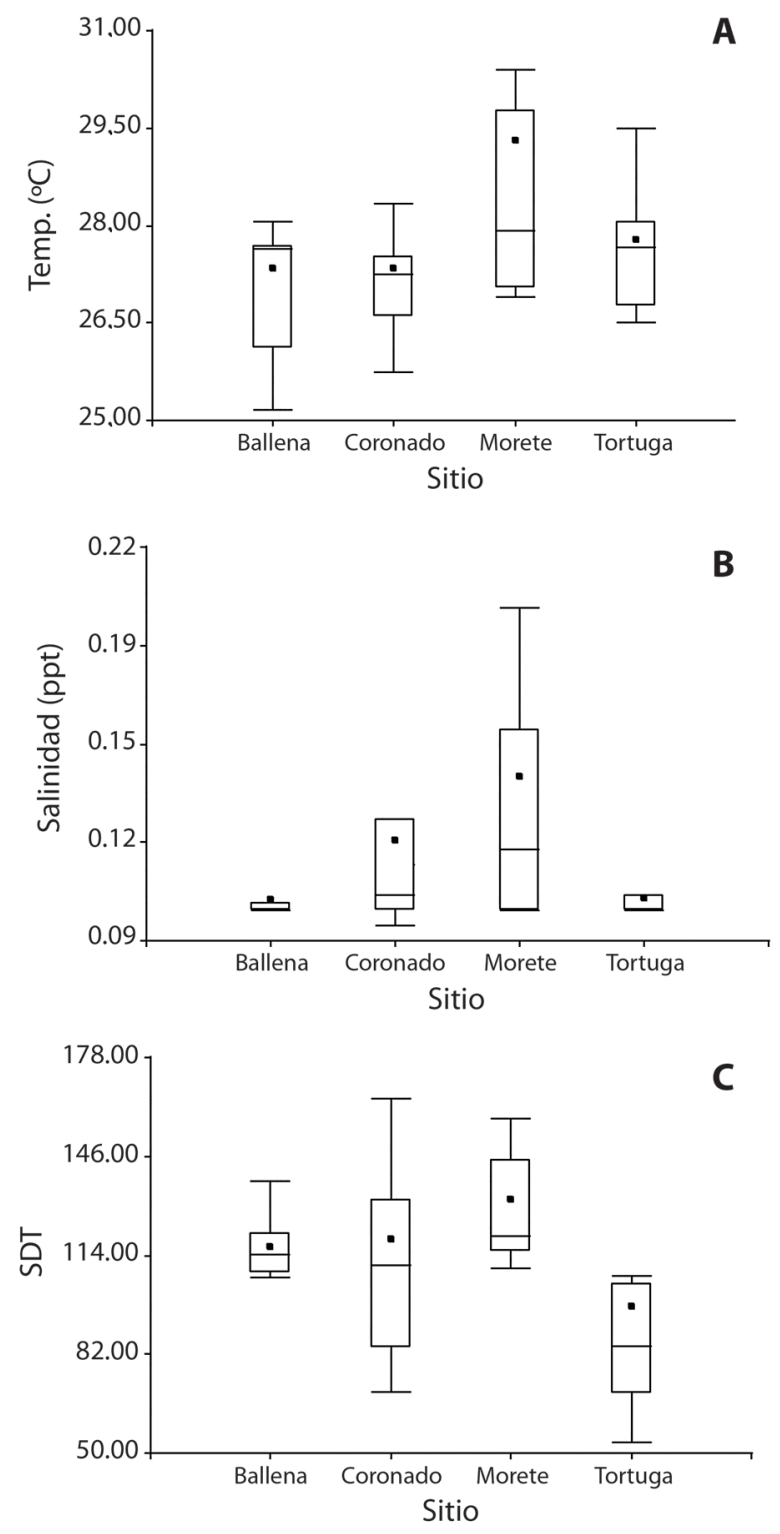

Fig. 3. Comportamiento variables temperatura, salinidad y sólidos disueltos totales por sitios de muestreo (medias, medianas y error estándar) entre junio 2017 y abril 2018.

En el caso de la turbidez, de las 22 muestras, 12 registraron condición cristalina, ocho semi-turbia, y dos con agua turbia (Fig. 5A), el registro Secchi de todas las muestras fue a profundidad de muestreo (visibilidad del disco alcanzó el fondo del cuerpo de agua). El ambiente rocoso se registró en los ríos Morete, Tortuga y la quebrada Ballena, sitio en el que se registró la mayoría de las ocasiones (Fig. 5B). Por su parte la corriente moderada fue la que prevaleció en los cuatro sitios de muestreo con más registros en el río Coronado $(n=4)$, la corriente muy lenta se registró en la quebrada Ballena y el río Morete, 

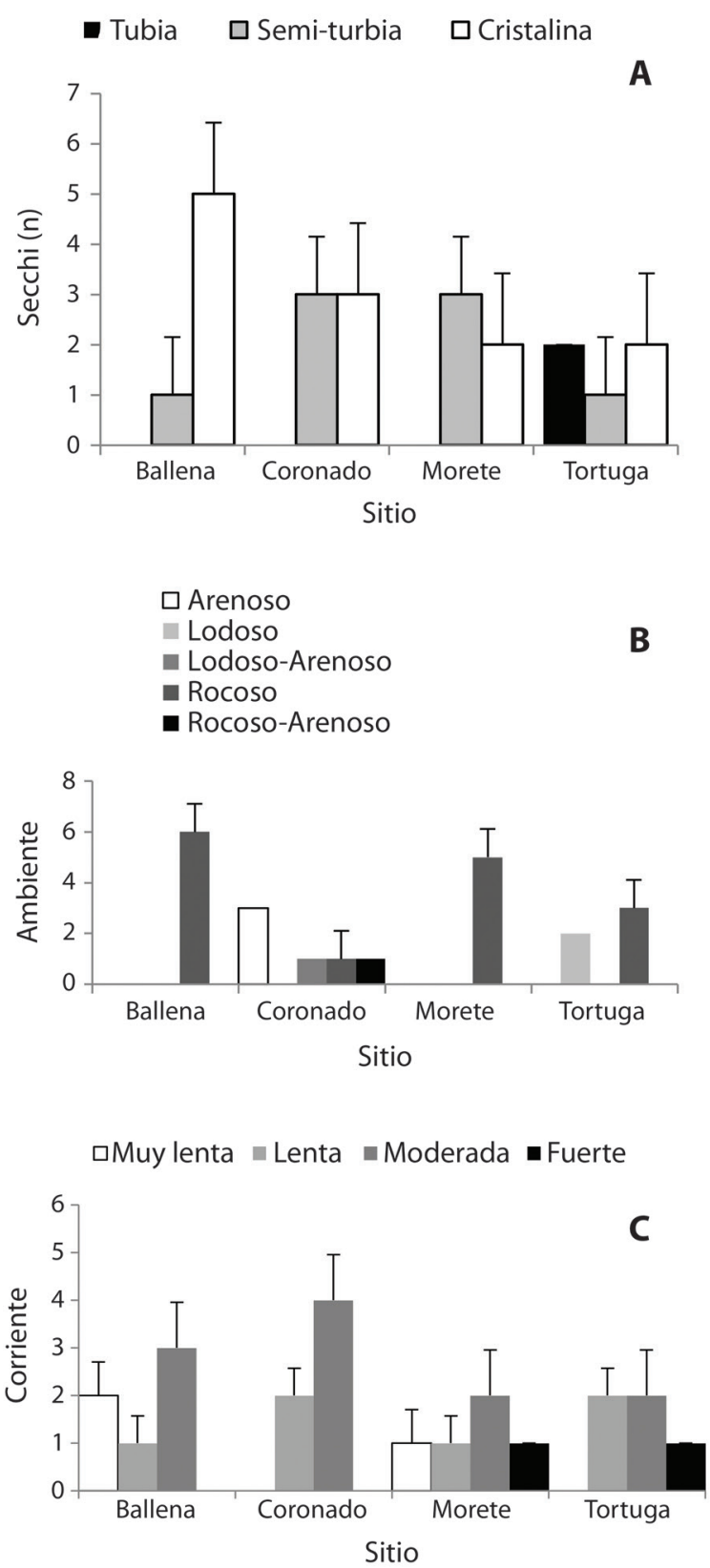

Fig. 5. Comportamiento variables turbidez, ambiente y corriente por sitios de muestreo (error estándar) entre junio 2017 y abril 2018. mientras que corrientes fuertes se registraron en una ocasión en los ríos Morete y Tortuga (Fig. 5C).

De acuerdo con el análisis multivariado de componentes principales, la ubicación en los ejes canónicos de los ríos Morete y Coronado, muestran alta correlación entre los mismos, además de alta correlación con variables como la temperatura (Morete $\lambda=29,30$ ), la salinidad (Morete $\lambda=0,14$; Coronado $\lambda=0,12$ ), las variables de oxígeno (Coronado $=$ OD $\lambda 10,53$; \%SAT $\lambda=133,55)$, por su parte la quebrada Ballena registró altas correlaciones con la turbidez $(\lambda=2,83)$, mientras que el río Tortuga fue influenciado, pero en menor grado por las variables de oxígeno $(\lambda=10,18)$ y la corriente $(\lambda=2,80)$ (Cuadro 1, Fig. 6).

\section{DISCUSIÓN}

Los resultados de la presente investigación muestran que estos cuatro cuerpos de agua evaluados según las variables consideradas, y a falta de otras como metales o materia fecal; tienen bajos niveles de contaminación o deterioro en su calidad de agua. Los elevados niveles de oxígeno registrados sugieren una reducida contaminación orgánica, con procesos de descomposición orgánica lentos y bajos. Como se sugiere en los resultados, se registró corrientes moderadas en la mayoría de los muestreos, lo cual promueve la sobre saturación de oxígeno en el agua.

Estos resultados son similares a los sugeridos por Umaña (1998) en varios ríos de la Península de Osa, incluso en momentos donde los ríos eran asediados por las agresivas actividades mineras de extracción de oro de placer en esa zona.

El oxígeno disuelto en el agua de un ecosistema fluvial natural es considerado una variable fundamental para la vida, y el registro de bajas concentraciones demuestran una alteración en la calidad medioambiental del mismo. De acuerdo con Boyd (1982) y Killgore y Hoover (2001) exposiciones prolongadas a bajas concentraciones de oxígeno disuelto en el agua (del orden de entre 1,4 mg/L a $3 \mathrm{mg} / \mathrm{L}$ ) generan alta mortalidad de los peces, situación lejana de ocurrencia en estos cuatro ríos evaluados, ya

CUADRO 1

Matríz de autovectores resultado de la correlación de las variables físico-químicas y los sitios de muestreo, Osa

\begin{tabular}{lcccccccccc}
\multicolumn{1}{c}{ Sitio } & $\begin{array}{c}\text { Altitud } \\
(\mathrm{msnm})\end{array}$ & OD $(\mathrm{mg} / \mathrm{L})$ & $\%$ SAT & ${ }^{\circ} \mathrm{C}$ & ppt & SDT & pH & $\begin{array}{c}\text { Turbidez } \\
\text { Secchi }\end{array}$ & Corriente & Ambiente \\
Ballena & 10,00 & 9,84 & 124,52 & 27,33 & 0,10 & 116,87 & 7,03 & 2,83 & 2,17 & 4,00 \\
Coronado & 10,00 & 10,53 & 133,55 & 27,34 & 0,12 & 119,15 & 7,03 & 2,50 & 2,67 & 2,50 \\
Morete & 10,00 & 10,04 & 130,52 & 29,30 & 0,14 & 132,27 & 6,96 & 2,40 & 2,60 & 4,00 \\
Tortuga & 5,00 & 10,18 & 130,24 & 27,77 & 0,10 & 97,32 & 6,92 & 2,00 & 2,80 & 3,20 \\
\hline
\end{tabular}




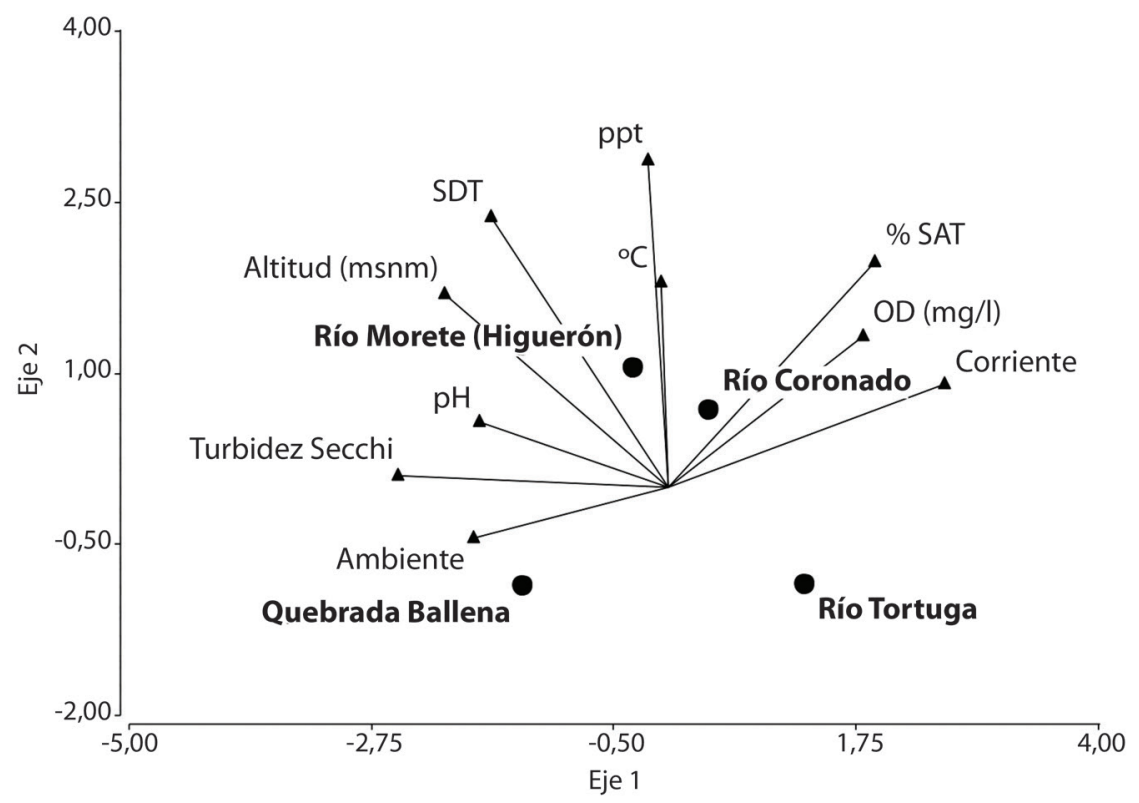

Fig. 6. Análisis de Componentes Principales a partir de las correlaciones parciales entre variables físico-químicas y sitios de muestreo $\left(\lambda_{1}=4,52 ; \lambda_{2}=3,00 ;\right.$ varianza acumulada $\left.=75 \%\right)$.

que los mismos presentaron concentraciones de oxígeno disuelto del orden de los $10 \mathrm{mg} / \mathrm{L}$.

Villegas (2011) determinó una relación en el aumento de la concentración de OD en el agua con respecto a la disminución del caudal en ríos que no se estancaron, situación también reportada por Velázquez-Velázquez, Vega-Cendejas y Navarro-Alberto (2008) en otras regiones del pacífico mesoamericano y por Calvo y Mora (2009) en otros ríos de Costa Rica con condiciones similares a los de la zona sur del país, lo cual también en la presente investigación podría sugerirse, como se muestra la alta correlación del OD y la corriente en el análisis de componentes principales, además del registro en su mayoría de corrientes muy lentas, lentas y moderadas.

La temperatura del agua osciló entre los 25 y $34^{\circ} \mathrm{C}$, registros similares a los de Umaña (1998) y Beita-Sandí y Barahona-Palomo (2010) en la Península de Osa, y de Villegas (2011) en otros ríos de los cantones de Osa, Golfito y Corredores. Estos valores son considerados como normales para la zona sur de Costa Rica (Winemiller \& Morales, 1989; Bussing, 2002; Rojas \& Rodríguez, 2008).

La salinidad en el agua fue una de las variables que más discrepó respecto a mediciones realizadas en otras investigaciones en la región. Villegas (2011) registró concentraciones de hasta 30ppt en ríos de Palmar Norte y Ciudad Neily, considerándose que estos ríos sugieren bajas concentraciones de $\mathrm{NaCl}$.
Son pocos los registros que se tienen de las concentraciones de sólidos (disueltos, suspendidos o sedimentables) para la zona sur del país. Velázquez-Velázquez, Vega-Cendejas y Navarro-Alberto (2008) al investigar algunos aspectos relevantes que determinan la variación espacio-temporal de peces en una laguna costera del pacífico mexicano, cuantificaron el máximo de sólidos disueltos totales en $28100 \mathrm{mg} / \mathrm{L}$, valor superior al determinado por Villegas en el 2011 en ríos de Osa, Golfito y Corredores zona sur de Costa Rica (19 213mg/L), lo que hace que los $173 \mathrm{mg} / \mathrm{L}$ como máximo registro de la presente en el río Coronado sea muy bajo, y evidencia las condiciones cristalinas que estos ríos mantuvieron en el periodo evaluado.

Lo anterior se confirma si se considera que Bonatti, Borge, Herrera y Paaby (2005) determinaron en 404t/ $\mathrm{km}^{2} /$ año la carga de sedimentos para la cuenca del río Grande de Térraba, sedimentos que se atribuyen a la combinación de un patrón de uso de la tierra con erosividad por precipitación. Por su parte Echeverría (2009) para la cuenca del río Rincón, estimó que el arrastre de sedimento fue de $105,45 \mathrm{t} / \mathrm{km}^{2} /$ año, registros que comparados ambos con las concentraciones de SDT de la presente investigación sugieren una carga de sedimentos muy baja en el periodo de estudio en los ríos Morete, Ballena, Tortuga y Coronado de Osa.

La fluctuación del pH entre 6,42 y 7,67 determinados en este estudio, también pueden considerarse normales 
al compararlos con los valores reportados por Winemiller y Morales (1989) en el Parque Nacional Corcovado $(6,2$ a 7,5); por Umaña (1998) de entre 7,75 y 8 en varios ríos de la Península de Osa; Rojas y Rodríguez (2008) en la cuenca del Térraba (6 a 8), Beita-Sandí y Barahona-Palomo (2010) para la cuenca del río Rincón $(6,62$ a 8,17) y Villegas (2011) en Palmar Norte y Ciudad Neily $(6,93$ y 8,59).

La condición general de "agua cristalina" en la mayoría de los muestreos es consistente con la baja concentración de sólidos disueltos totales detectados, no obstante según Birtwell 1999, Henley, Patterson, Neves y Lemly (2000) y Berry, Rubinstein, Melzian y Hill (2003) se carece de estudios que comprueben correlaciones entre las NTU (Unidades Nefelométricas de Turbidez) -unidad de turbidez-y los mg/L -unidad de concentración de sólidos- debido a que la turbidez puede estar influenciada por diversos factores (nutrientes, sedimentos, microorganismos, minerales, entre otros) mientras que los sólidos son una relación peso-volumen físicamente establecida.

Los ríos son ecosistemas acuáticos sumamente dinámicos, y son influenciados por todas las condiciones ambientales circundantes, tanto a nivel local o focalizado como en el rango de acción de una gran cuenca hidrográfica. Son muchos los factores que pueden afectar las condiciones físicas y químicas del agua de un río o una quebrada, no obstante a partir de las mediciones sobre variables de esta calidad del agua podría determinarse su estado de conservación o de degradación, en un momento y lugar determinado (Hernández, Martínez, Moreno, \& Martínez, 2012).

A partir de las mediciones realizadas al agua de estos cuatro cuerpos superficiales en los distritos de Bahía Ballena y Puerto Cortés del cantón de Osa, en la zona sur de Costa Rica, se puede inferir que durante el periodo evaluado de junio 2017 a abril 2018 las condiciones físico-químicas de la misma son similares para los cuatro sitios.

No hay evidencia estadística $(p \geq 0,05)$ que sugiera que la relativa mejor condición de las zonas de protección en el distrito de Bahía Ballena genere una mejor calidad del agua en los ríos de la región, no obstante el distrito de Puerto Cortés ha tenido mayor deterioro de sus recursos naturales por los cultivos extensivos de banano en el pasado o de arroz y palma aceitera en el presente, por lo que se descarta esta hipótesis y se tiene que los ríos y quebradas en el cantón de Osa, en la zona sur de Costa Rica tienen condiciones de calidad de agua similares.

\section{AGRADECIMIENTOS}

Agradezco a Maurizio Protti Quesada por ser tutor y maestro durante mi vida académica y profesional, a la Universidad Nacional por permitirme desarrollar este proyecto de estudios doctorales en su programa DOCINADE, y al Área de Conservación Osa del SINAC por brindar los permisos para esta investigación y el apoyo técnico de sus funcionarios.

\section{REFERENCIAS}

Aguirre, M.R., Vanegas, E.A., \& García, N. (2016). Aplicación del Índice de Calidad del Agua (ICA); Caso de estudio: Lago de Izabal, Guatemala. Revista Ciencias Técnicas Agropecuarias, 25(2), 39-43.

Balzarini, M.G., Casanoves, F., Di Rienzo, J.A., González, L.A., Robledo, C.W., \& Tablada, E.M. (2017). InfoStat, Manual del Usuario (versión 1.1., $1^{\text {era }}$ ed.). Grupo InfoStat, FCA, Universidad Nacional de Córdoba: Editorial Brujas Argentina.

Beita-Sandí, W., \& Barahona-Palomo, M. (2010). Físico-química de las aguas superficiales de la cuenca del río Rincón, Península de Osa, Costa Rica. UNED Research Journal, 2(2), 157-179. DOI: 10.22458/urj.v2i2.156

Berry, W., Rubinstein, N., Melzian, B., \& Hill, B. (2003). The biological effects of suspended and bedded sediment (SABS) in aquatic systems: A review internal report. U.S.A.: Environmental Protection Agency.

Birtwell, I.K. (1999). The effects of sediment on fish and their habitat. Ottawa, Canada: Fisheries and Oceans, Canadian Stock Assessment Secretariat.

Bonatti, J., Borge, C., Herrera, B., \& Paaby, P. (2005). Efectos ecológicos del cultivo de la piña en la cuenca media del río General-Térraba de Costa Rica (Informe Técnico sin publicación). San José, Costa Rica:TNC.

Boyd, C.E. (1982). Water quality management for pond fish culture. Amsterdam: Elsevier Scientific Pub. Co.

Bussing, W.A. (2002). Peces de las aguas continentales de Costa Rica. San José, Editorial de la Universidad de Costa Rica.

Calvo, G., \& Mora, J. (2009). Evaluación y clasificación preliminar de la calidad del agua de las cuencas de los ríos Tárcoles y Reventazón. Parte IV: Análisis estadístico variables relacionadas con la calidad del agua. Revista Tecnología en Marcha, 22(1), 57-64.

Calvo, G., \& Mora, J. (2012). Análisis de la calidad de varios cuerpos de agua superficiales en el GAM y la Península de Osa utilizando el Índice Holandés. Revista Tecnología en Marcha, 25(5), 37-44. DOI: 10.18845/tm.v25i5.471 
Calvo-Brenes, G., \& Mora-Molina, J. (2015). Evaluación de la calidad del agua en los ríos Tigre y Rincón de la península de Osa en dos periodos de tiempo distintos. Revista Tecnología en Marcha, 28(3), 55-63. DOI: 10.18845/ tm.v28i3.2411

Correa-Araneda, F., Rivera, R., Urrutia, J., De Los Ríos, P., Contreras, A., \& Encina- Montoya, F. (2010). Efectos de una zona urbana sobre la comunidad de macroinvertebrados bentónicos de un ecosistema fluvial del sur de Chile. Limnetica, 29(2), 183-194.

Echeverría, S. (2009). Uso de la tierra y transporte de sólido en suspensión en la cuenca del río Rincón, Península de Osa, Puntarenas, Costa Rica (Tesis de posgrado). Universidad Estatal a Distancia, San José, Costa Rica.

Flores-Stulzer, E., Villalobos-Sandí, N., Piedra-Castro, L., \& Scholz, C. (2017). Evaluación breve de la presencia de diatomeas y su relación con algunos parámetros físico-químicos en el río Pirro, Heredia, Costa Rica. Uniciencia, 31(2), 99-109. DOI: 10.15359/ru.31-2.7

Fowler, J., Cohen, L., \& Jarvis, P. (1998). Practical statistics for field biology ( $2^{\text {nd }}$ ed.). West Sussex, England: John Wiley \& Sons.

Glaser, U., \& Glaser, W. (1996). Aqualog reference fish of the world: South American Cichlids. Germany: Editorial A.C.S. $\mathrm{GmbH}$.

Henley, W.E., Patterson, M.A., Neves, R.J., \& Lemly, A.D. (2000). Effects of sedimentation and turbidity on lotic food webs: A conscise review for natural resource managers. Reviews in Fisheries Science, 8(2), 125-139. DOI: 10.1080/10641260091129198

Hernández, A.I., Martínez, R., Moreno, D., \& Martínez, L. (2012). Diversidad de insectos acuáticos como bioindicadores de la calidad del agua de la microcuenca del río Jutiapa en las quebradas Corralitos, Limones y Jutiapa del parque nacional La Tigra, Francisco Morazán, Honduras. Revista Ciencia y Tecnología, 10, 25-56.

Killgore, K.J., \& Hoover, J.J. (2001). Effects of hypoxia on fish assemblages in a vegetated waterbody. Journal of Aquatic Plant Management, 39, 40-44.

MINAE (Ministerio del Ambiente y Energía). (2000). Estrategia nacional de conservación y uso sostenible de la biodiversidad. San José, Costa Rica.

Ocón, C., \& Rodriguez, A. (2004). Presence and abundance of Ephemeroptera and Other sensitive macroinvertebrates in relation with habitat conditions in pampean streams (Buenos Aires, Argentina). Archiv für Hydrobiologie, 159, 473-487. DOI: 10.1127/0003-9136/2004/0159-0473

Parreño, D., Bernal, P. \& Palmer, J. (2015). Ríos saludables de Osa: una novedosa red de seguimiento de cuerpos de agua por parte de la comunidad. Cuadernos de Biodiversidad, 49, 14-19.
Posada, J., Roldán, A., \& Ramírez, J. (2000). Caracterización fisicoquímica y biológicas de la calidad de aguas de la cuenca de la quebrada Piedras Blancas, Antioquia, Colombia. Revista de Biología Tropical, 48, 59-70.

Protti, M., Sáenz, I., Guevara, M., \& Herrera, M. (2005a). Evaluación de la ictio y entomofauna acuática en el área de impacto y zonas aledañas al Proyecto Hidroeléctrico Cariblanco (Informe Científico Técnico Periodo 2004). Heredia, Costa Rica: Laboratorio de Recursos Naturales y Vida Silvestre, Universidad Nacional.

Protti, M., Sáenz, I., Guevara, M., \& Herrera, M. (2005b). Evaluación de la ictio y entomofauna acuática en el área de impacto y zonas aledañas al Proyecto Hidroeléctrico Cariblanco (Informe Científico Técnico Anual: Periodo Diciembre 2004-Octubre 2005). Heredia, Costa Rica: Laboratorio de Recursos Naturales y Vida Silvestre, Universidad Nacional.

Protti, M., Sáenz, l., Guevara, M., \& Herrera, M. (2007). Evaluación de la ictio y entomofauna acuática en el área de impacto y zonas aledañas al Proyecto Hidroeléctrico Cariblanco (Informe Científico Técnico Anual: Periodo Diciembre 2005-Octubre 2006). Heredia, Costa Rica: Laboratorio de Recursos Naturales y Vida Silvestre, Universidad Nacional.

Ramírez, A., \& Pringle, C. (1998). Invertebrate drift and benthic community dynamics in a lowland neotropical stream, Costa Rica. Dynamics in a lowland neotropical stream, Costa Rica. Hydrobiología, 386, 19-26. DOI: 10.1023/A:1003409927131

Rojas, R., \& Rodríguez, O. (2008). Diversidad y abundancia ictiofaunística del río Grande de Térraba, sur de Costa Rica. Revista de Biología Tropical, 56(3), 1429-1447.

SINAC (Sistema Nacional de Áreas de Conservación). (2007). Grúas II. Análisis de vacíos de conservación en Costa Rica: Vol II. Análisis de Vacíos en la Representatividad e Integridad de la Biodiversidad de los sistemas de aguas continentales ( $1^{\text {a }}$ ed.). San José, Costa Rica: Asociación Conservación de la Naturaleza.

Sokal R., \& Rohlf, J. (1981). Biometry. New York, USA: Freeman and Company.

Umaña, G. (1998). Characterization of some Golfo Dulce drainage basin rivers (Costa Rica). Revista de Biología Tropical, $46(6), 125-135$.

Velázquez-Velázquez, E., Vega-Cendejas, M., \& Navarro-Alberto, J. (2008). Spatial and temporal variation of fish assemblages in a coastal lagoon of the Biosphere Reserve La Encrucijada, Chiapas, Mexico. Revista de Biología Tropical, 56(2), 557-574.

Villegas, J.C. (2011). Relación entre la diversidad de ictiofauna y la calidad del agua en ríos con diferente grado de afectación por diques y canales en la zona sur de Costa Rica (Tesis de posgrado). Universidad Estatal a Distancia, San José, Costa Rica. 
West, D.W., Boubée, J., \& Barrier, R.F.G. (1997). Responses to $\mathrm{pH}$ of nine fishes and one shrimp native to New Zealand freshwaters. New Zealand Journal of Marine and Freshwater Research, 31, 461-468. DOI: 10.1080/00288330.1997.9516779
Winemiller, K.O., \& Morales, N.E. (1989). Comunidades de peces del Parque Nacional Corcovado luego del cese de las actividades mineras. Brenesia, 31, 75-91. 